\title{
Lung nodules: size still matters
}

\author{
Anna Rita Larici, Alessandra Farchione, Paola Franchi, Mario Ciliberto, \\ Giuseppe Cicchetti, Lucio Calandriello, Annemilia del Ciello and \\ Lorenzo Bonomo
}

Number 4 in the Series "Radiology" Edited by Nicola Sverzellati and Sujal Desai

Affiliation: Institute of Radiology, Catholic University of the Sacred Heart, Fondazione Policlinico Universitario A. Gemelli, Rome, Italy.

Correspondence: Anna Rita Larici, Institute of Radiology, Catholic University of the Sacred Heart, Fondazione Policlinico Universitario A. Gemelli, Largo F. Vito 1, 00168 Rome, Italy. E-mail: annarita.lariciđunicatt.it

@ERSpublications

Size and growth rate remain the main determinants of nodule management http://ow.ly/mtDB30gugUg

Cite this article as: Larici AR, Farchione A, Franchi P, et al. Lung nodules: size still matters. Eur Respir Rev 2017; 26: 170025 [https://doi.org/10.1183/16000617.0025-2017].

ABSTRACT The incidence of indeterminate pulmonary nodules has risen constantly over the past few years. Determination of lung nodule malignancy is pivotal, because the early diagnosis of lung cancer could lead to a definitive intervention. According to the current international guidelines, size and growth rate represent the main indicators to determine the nature of a pulmonary nodule. However, there are some limitations in evaluating and characterising nodules when only their dimensions are taken into account. There is no single method for measuring nodules, and intrinsic errors, which can determine variations in nodule measurement and in growth assessment, do exist when performing measurements either manually or with automated or semi-automated methods. When considering subsolid nodules the presence and size of a solid component is the major determinant of malignancy and nodule management, as reported in the latest guidelines. Nevertheless, other nodule morphological characteristics have been associated with an increased risk of malignancy. In addition, the clinical context should not be overlooked in determining the probability of malignancy. Predictive models have been proposed as a potential means to overcome the limitations of a sized-based assessment of the malignancy risk for indeterminate pulmonary nodules.

Introduction: the "size" of the problem

By definition, a lung nodule is a rounded or irregular opacity, which may be well or poorly defined, measuring $\leqslant 3 \mathrm{~cm}$ in diameter, surrounded by aerated lung on radiological imaging [1]. The definition includes nodules in contact with pleura and excludes those associated with lymphadenopathies or pleural disease [2]. An opacity $<3 \mathrm{~mm}$ should be referred to as a micronodule [1].

With the introduction of multidetector computed tomography (MDCT), the number of detected lung nodules, particularly those small in size, has dramatically increased. The prevalence of noncalcified lung

Received: March 172017 | Accepted after revision: Oct 282017

Previous articles in this series: No. 1: Walsh SLF. Multidisciplinary evaluation of interstitial lung diseases: current insights. Eur Respir Rev 2017; 26: 170002. No. 2: Elicker BM, Kallianos KG, Henry TS. The role of high-resolution computed tomography in the follow-up of diffuse lung disease. Eur Respir Rev 2017; 26: 170008. No. 3: Robbie H, Daccord C, Chua F, et al. Evaluating disease severity in idiopathic pulmonary fibrosis. Eur Respir Rev 2017; $26: 170051$.

Conflict of interest: None declared.

Provenance: Commissioned article, peer reviewed.

Copyright CERS 2017. ERR articles are open access and distributed under the terms of the Creative Commons Attribution Non-Commercial Licence 4.0. 
nodules has been reported as 33\% (range 17-53\%) and 13\% (range 2-24\%), in a screening and nonscreening study population, respectively [2]. Notably, screening studies include asymptomatic subjects at high risk of developing lung cancer, among whom the majority have small noncalcified lung nodules on thin-section MDCT [3], while in a nonscreening population a lung nodule represents an incidental finding.

Since the increase in the detection rate of small pulmonary nodules, the clinical significance of these findings represents a new challenge $[2,4]$, and the optimal management of each case becomes pivotal and should be conducted according to the clinical setting.

After detecting a lung nodule, the main goal for physicians is to identify a nodule suspicious enough to warrant further testing as early as possible, but avoiding unnecessary diagnostic or therapeutic procedures. In cases of malignant nodules, the early diagnosis of lung cancer could provide a safe and definitive solution. In this context, detection and follow-up using computed tomography (CT) play an important role, even though the risk of false-positive results, as well as the biological cost in terms of radiation burden from several CT scans required during follow-up and healthcare costs should all be taken into account [4].

Nodule size and growth rate remain the most widely used predictors to assess probability of nodule malignancy and to determine nodule management according to the international guidelines [2, 4-7]. Indications included in the guidelines are based on the existence of a directly proportional relationship between the initial size, growth rate and risk of malignancy of nodules.

Until now, nodule management has been based on the measurement of nodule diameter, even though the more recent guidelines introduced nodule volume as an indicator. The British Thoracic Society (BTS) added initial volume and volume doubling time (VDT) calculations to the diameter, and the Fleischner Society added volume [2,7]. The data on volumetry are mainly derived from the Dutch-Belgian Lung Cancer Screening trial (NELSON) evidence [8].

When considering size for managing an indeterminate pulmonary nodule the existence of a potential inherent inaccuracy of nodule measurements in terms of diameter, volume and growth rate should be taken into account.

In this review we debate the relevance of size and growth rate in nodule characterisation, as well as the currently used methods for measuring pulmonary nodules, their limitations and factors influencing nodule measurement variations and growth estimation. Special considerations on subsolid nodules (SSNs) are included in this context. Finally, the risk prediction models that integrate clinical and nodule characteristics besides size and the role of nodule size as a factor affecting the critical time for follow-up are briefly discussed.

\section{Size and malignancy}

In the above-described scenario, a strong effect of the nodule size on predicting malignancy has been underlined, even though the management of a pulmonary nodule cannot solely rely on size.

It has been widely demonstrated that there is a proportional increase in the risk of malignancy as the nodule diameter increases, as reported in an extensive review [9]. McWilliams et al. [10] confirmed the observation that nodule diameter is associated with lung cancer probability, with a significant nonlinear relationship in patients undergoing low-dose CT screening $(\mathrm{p}<0.001$ for nonlinearity).

It is worth noting that the prevalence of malignancy in nodules measuring $<5 \mathrm{~mm}$ is very low, ranging between 0 and $1 \%[8,9]$. In the National Lung Screening Trial (NLST), the prevalence of lung cancer among patients with 4-6-mm nodules was very low: $0.49 \%$ (18 out of 3668 patients) at baseline, $0.3 \%$ (12 out of 3882 patients) in the first screening round and $0.7 \%$ (15 out of 2023 patients) in the second round of screening [11, 12]. Moreover, in the NELSON study malignancy risk in subjects with nodules measuring $<5 \mathrm{~mm}$ or $<100 \mathrm{~mm}^{3}$ was similar to the risk in subjects without nodules [8]. By taking into account these observations, according to the recent guidelines the nodule size threshold (diameter or volume) for determining the need for follow-up has been increased to $5 \mathrm{~mm}$ or $80 \mathrm{~mm}^{3}$ for BTS guidelines and $6 \mathrm{~mm}$ or $100 \mathrm{~mm}^{3}$ for Fleischner Society guildeines [2, 7].

In table 1 we summarise the relationships between the diameter of pulmonary nodules and the prevalence of malignancy, as reported in a large literature review [9], and between diameter, volume and VDT with the prevalence of malignancy as reported in the NELSON screening study by HoREwEG et al. [8].

Apart from nodule size, it is well known that nodule appearance in terms of density affects the probability of malignancy, reflecting histological differences between lesions. 
TABLE 1 Relationship between nodule size, expressed as diameter and volume, and growth rate, expressed as volume doubling time (VDT), with the prevalence of malignancy

Prevalence of malignancy \%

NELSON screening study [8] Literature review [9]

\begin{tabular}{lcc}
\hline Diameter mm & & \\
$<5$ & 0.4 & $0-1$ \\
$5-10$ & 1.3 & $6-28$ \\
$\geqslant 10$ & 15.2 & $64-60$ \\
$\geqslant 20$ & & \\
Volume $\mathbf{m m}^{3}$ & & \\
$\leqslant 100$ & 0.6 & \\
$100-300$ & 2.4 & \\
$\geqslant 300$ & 16.9 & \\
VDT days & 0.8 & \\
$\geqslant 600$ & 4 & \\
$400-600$ & 9.9 & \\
$\leqslant 400$ & & \\
\hline
\end{tabular}

Data from $[8,9]$.

Data from the literature confirmed the above-described relationship between nodule size and malignancy even when distinguishing lung nodules according to their density. The first screening trials demonstrated a $\leqslant 1 \%$ malignancy risk in solid nodules $<5 \mathrm{~mm}$ in diameter, as reported in the Early Lung Cancer Screening Project (ELCAP), and in the Mayo Clinic CT screening trial the majority (80\%) of cancers were $>8 \mathrm{~mm}$ in diameter [13-15].

Regarding SSNs, including pure ground-glass nodules ( $p$ GGNs), named nonsolid nodules and part-solid nodules (PSNs), results derived from the ELCAP [14] and the following I-ELCAP screening studies [16, 17] demonstrated a prevalence of malignancy for small nodules of $0 \%$ (considering a maximum nodule diameter of $5 \mathrm{~mm}$ ) and $<1 \%$ (considering a maximum nodule diameter of $6 \mathrm{~mm}$ ). In both experiences an increase in malignant cases was associated with an increase in nodule diameter [14, 16, 17]. Furthermore, in the early ELCAP screening the prevalence of malignancy was higher among SSNs, particularly when considering PSNs (18\% for pGGNs and 63\% for PSNs), than among solid ones (7\%) [14].

Therefore, it has been suggested that for SSNs, management and T staging assessment, as included in the tumour node metastasis classification, should be adjusted by measuring both the overall nodule size and the solid component size $[6,18,19]$.

\section{Size changes: the growth rate}

Small nodules are not reliably characterised by contrast enhancement evaluation or positron emission tomography scanning and biopsy is difficult to perform on these nodules. However, the risks involved in a surgical diagnosis would be excessive compared to the relatively low prevalence of malignancy in the small nodules.

Nodule growth, determined by imaging surveillance, could be used as a diagnostic tool for assessing malignancy [5]. By performing an "early" repeated CT within 30 days, YANKELEviTZ et al. [20] accurately detected growth in nodules as small as $5 \mathrm{~mm}$ and ZHAO et al. [21] demonstrated that the majority of resolving nodules disappeared at the same time point.

ColLins et al. [22] advanced the theory of an exponential growth of tumours to predict the growth rate, which assumes a uniform three-dimensional (3D) tumour increase. Therefore, growth is typically expressed in terms of VDT, defined as the time taken for the nodule to double in volume or to increase $26 \%$ in diameter $[5,22]$.

LiNDELL et al. [23] analysed the growth curves of lung cancer detected in a screening population, observing that lung cancers may be associated with a fairly steady or accelerated growth, particularly the more aggressive tumours. The authors concluded that the initial tumour size at one point and the interval growth assessed between two points are not predictive of the future growth, therefore the likelihood of a nodule to be malignant may be misinterpreted by using models assuming an exponential growth [23].

Interesting results have been reported on VDT by Xu et al. [24], who retrospectively investigated the role of morphological features, size and VDT in the differentiation between benign and malignant lung solid 
nodules detected in the NELSON trial. The study demonstrated that by using a multivariate model, when follow-up data are available, nodule growth assessed by VDT at 1-year follow-up was the only strong predictor for malignancy.

A wide range of growth rates for lung cancer has been reported in literature, according to different methods used to measure the nodule (diameter, manual bidimensional or automated 3D volume), as well as to the histological subtypes and radiological appearance [2]. The clinical setting seems not to affect the nodule growth rate, in fact no significant differences between screening and nonscreening studies have been demonstrated in this regard [25].

VDTs in the range of 20-400 days have been reported for malignant solid nodules, with a $98 \%$ negative predictive value of malignancy for a VDT of $>500$ days (calculated using volumetric software) [26]. Longer times have been considered for malignant SSNs, in particular $813 \pm 375$ days and $457 \pm 260$ days for pGGNs and PSNs, respectively [2, 25-30]. Notably PSNs with a solid component $\leqslant 5 \mathrm{~mm}$ showed significantly longer VDT, compared to lesions with a solid portion $>5 \mathrm{~mm} \mathrm{[31].}$

In the NELSON screening trial, growing nodules were stratified in risk groups according to VDT (high risk <400 days; intermediate risk 400-600 days; low risk >600 days) [32]. Specifically, VDT stratified the probabilities of malignancy as follows: $0.8 \%$ (95\% CI $0.4-1.7 \%$ ) for VDT $\geqslant 600$ days, $4.0 \%$ (95\% CI $1.8-$ 8.3\%) for VDT 400-600 days and 9.9\% (95\% CI 6.9-14.1\%) for VDT $\leqslant 400$ days [32].

\section{Size measurements: methods and limitations}

Size measurements of lung nodules need to be accurate and precise to allow correct risk classification and to assess changes in nodule size over time. Accuracy describes the difference between the mean value of the object measured and its true value [33]. Precision refers to variability in performing different measurements on the same experimental unit, when measurement setting is either stable or variable [33]. These characteristics are particularly relevant for small-sized nodules whose changes, even when doubled in time, are difficult to recognise visually.

Lung nodules can be evaluated according to diameter, area or volume, calculated either by manual or semi-automated/automated methods. Semi-automated methods allow the operator manual interaction with the automated modality.

Results from the literature agree that volume measurement is a method with a better performance in nodule sizing, as well as in assessing nodule's growth [34, 35]. MентA et al. [36] added volumetric nodule measurement to an existing prediction model for nodule malignancy estimation, showing an increase in the number of nodules correctly classified. Notably, the study included only lesions $<15 \mathrm{~mm}$ in diameter. A more recent study on lung cancer probability applied to the NELSON population compared nodule management strategies based on nodule volume (cut-offs $100 \mathrm{~mm}^{3}$ and $300 \mathrm{~mm}^{3}$ for an indeterminate and a positive test, respectively) versus nodule diameter (cut-offs $5 \mathrm{~mm}$ and $10 \mathrm{~mm}$ for an indeterminate and a positive test, respectively) [37]. The study concluded that the volume-based analysis had a sensitivity and negative predictive value comparable to those resulting from the diameter-based analysis, whereas the specificity and positive predictive values were higher [37].

In this context technical and practical issues need to be considered. Firstly, nodule diameter measurement is not a reliable method for assessing the entire nodule dimension and it is affected by non-negligible inter- and intra-observer variability. Secondly, volume measurement methods tend to be more susceptible to the influence of technical parameters and software type used to perform volumetry. Moreover, automated systems are not routinely used, mainly because they usually are not integrated in the picture archiving and communication system [38] and their application may be time consuming.

\section{One-dimensional and two-dimensional measurements}

The most commonly used method to define nodule size consists in measuring the maximum nodule diameter using a one-dimensional (1D) calliper, according to the RECIST (Response Evaluation Criteria in Solid Tumours) criteria [39]. This method has been promoted as a more practical and simple system than that of the World Health Organization [39]. Established in the late 1970s, the latter relies on two-dimensional (2D) or cross-sectional area measurement, calculated by multiplying the tumour's maximum diameter in the transverse plane by its largest perpendicular diameter on the same image [39]. Interestingly, the $2 \mathrm{D}$ measurement showed a greater variability when applied to solid nodules compared to 1D and volumetric methods [40]. Moreover, as reported by Jennings et al. [34], in the assessment of growth the use of the cross-sectional area did not perform significantly better than the diameter.

Another method of measuring nodule size is to assess the average diameter, calculated between the maximal long-axis and perpendicular maximal short-axis diameters assessed on transverse CT sections. 
This method has been recommended by the Fleischner Society in the guidelines published in 2005 and 2013 for management of indeterminate pulmonary nodules, as it reflects the entire nodule dimensions more accurately $[4,6]$.

There are some limitations of these methods affecting both accuracy and precision of nodule measurements. When using $1 \mathrm{D}$ or $2 \mathrm{D}$ measurements we consider only the subset of data included in the maximum cross-sectional diameter or area measured on the axial image [41]. It is worth noting that the maximum nodule diameter may be in nonaxial images (figure la and b).

Errors and variability are particularly evident when considering small nodules. In a retrospective analysis including only solid noncalcified pulmonary nodules $<2 \mathrm{~cm}$ in diameter, Revel et al. [42] stated that the largest transverse cross-sectional nodule diameter manually measured by positioning an electronic calliper is not reliable due to a poor intra- and inter-reader agreement (figure $1 \mathrm{c}$ and $\mathrm{d}$ ). The best intra-reader repeatability coefficient (5\% error rates) was 1.32 and the $95 \%$ limits of agreement for the difference among readers was \pm 1.73 [42]. From a clinical point of view, this means that by using the $1 \mathrm{D}$ method, measurement values $<1.32$ and $<1.73 \mathrm{~mm}$ cannot be distinguished from errors.
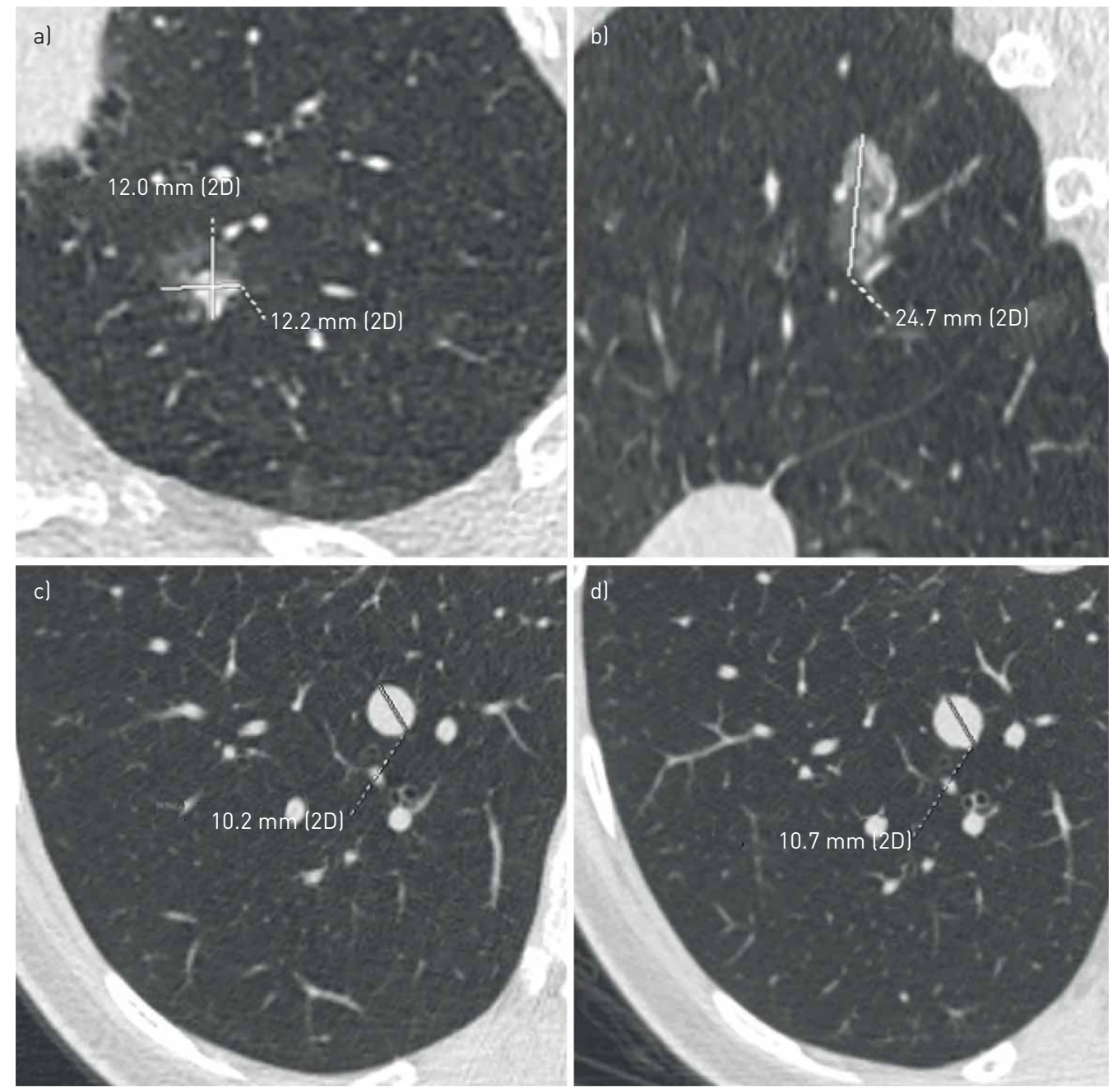

FIGURE 1 Limitations of two-dimensional (2D) measurements. The axial diameter may not be the maximum one in the evaluation of lung nodules. a) A small part-solid nodule in the apico-posterior segment of the left upper lobe, with a maximum axial diameter of $12 \times 12.2 \mathrm{~mm}$; b) the sagittal multiplanar reconstruction shows that the largest diameter of the same nodule is the sagittal one of $24.7 \mathrm{~mm}$. The multiplanar evaluation of nodule diameter is especially important to document asymmetrical growth of nodules. c), d) The low level of agreement when measuring small nodules: for the same nodule in the right lower lobe two different diameter values have been reported by two readers. Considering the nearest whole diameter of the two values, it results in $1 \mathrm{~mm}$ difference in the maximum diameter, a significant difference when considering small nodules. 
Furthermore, a study derived from NLST demonstrated that variations in 1D measurement of pulmonary nodule diameter performed using electronic calliper account for much of the disagreement among readers in the classification of the screening results as positive or negative, in particular when considering nodules with irregular shape and indistinct margins [43].

In the attempt to reduce variability in nodule measurements, the latest version of the Fleischner Society guidelines published in 2017 recommended the calculation of the average nodule diameter between the long and the short axis in whichever plane (axial, coronal or sagittal) the nodule shows its maximum dimension [7]. A following statement focused on recommendations for measuring pulmonary nodules clarified that for nodules $<1 \mathrm{~cm}$ the dimension should be expressed as average diameter, while for larger nodules both short- and long-axis diameters taken on the same plane should be reported [44].

With regard to SSNs, visual evaluation is a difficult task as nodule margins tend to be ill-defined and have a low contrast with respect to the surrounding lung parenchyma. In this context, uncertainties exist not only in the nodule measurement, due to difficulties in delineating nodule margins and different densitometric components of PSNs, but also in the classification of nodule morphological characteristics (i.e. $\mathrm{pGGN}$ or PSN) $[45,46]$.

For SSNs a maximum variability of $\pm 2.2 \mathrm{~mm}$ in measuring both the longest nodule diameter and the average one has been reported [46]. The recent BTS guidelines corroborated these data and stated that for SSNs an increase in the maximum diameter $\geqslant 2 \mathrm{~mm}$ is strongly predictive of malignancy [2]. When considering small SSNs $(<1 \mathrm{~cm})$ the variability in measuring nodule dimension was lower when using the average diameter than the longest one [46]. The latest statement from the Fleischner Society on nodule measurements supports this evidence and recommends the expression of the dimension of SSNs $<1 \mathrm{~cm}$ as average diameter, as for solid nodules [44].

Two recent studies focused on the evaluation of observer variability in visual classification of SSNs and the potential implication on patient management, both in a screening and nonscreening setting [45, 47]. Agreement values were moderate (intra- and inter-observer agreement $\kappa$-values of 0.57 and 0.51 , respectively in the screening setting; inter-observer agreement $\kappa$-value of 0.56 in the nonscreening setting) and discordance in nodule classification was mainly due to the assessment of the solid component, in terms of presence and size $[45,47]$. This variability is probably related to the lack of standardised criteria on how to measure different densitometric components of SSNs and on which CT window setting (i.e. lung or mediastinal) should be used, at the time of their publication.

A recent article demonstrated that the lung window setting has a comparable reproducibility, but higher accuracy in SSN classification and measurement of the solid component than the mediastinal window setting [48]. Moreover, LEE et al. [49] showed that the size of a solid portion displayed at the lung window setting better correlates with the nodule invasive component. Conversely, by using a mediastinal window setting, only areas $>-160$ Hounsfield units can be detected as solid, resulting in an underestimation of the size of the solid portion (figure 2) [45, 46]. Therefore, on the basis of the updated literature, recommendations from the Fleischner Society suggest the use of the lung window setting and the high spatial frequency (sharp) filter to judge the presence of a solid component, and the measurement of both the solid and nonsolid portions in a PSN. The same display window setting is recommended for measuring solid nodules [44].

\section{0 measurements}

The most commonly reported 3D methods for nodule volume measurement are those performed using manual or semi-automated/automated techniques.

When measuring volume manually, the region of interest (ROI) is first defined by outlining the 2D nodule borders section by section and then applying $3 \mathrm{D}$ software that estimates nodule volume from the number of voxels included within the multiple ROIs [50].

By using semi-automated/automated methods the ROI is defined automatically or by starting from a point inside the nodule selected by the user. Afterwards a segmentation algorithm is applied to outline 3D nodule borders and calculate the volume. Segmentation is often based on a threshold density technique followed by voxel counting for the volume estimation. Alternative methods include the estimation of the nodule shape in the continuous space of the object [50].

One of the first applications of volumetric analysis was the study by YANKELEVITZ et al. [41], who compared the accuracy of $3 \mathrm{D}$ techniques in determining volume with the accuracy of $2 \mathrm{D}$ techniques in defining a cross-sectional area. On synthetic spheres volume estimation was reliable as the area measurement and, moreover, the VDT estimated on in vivo nodules appeared to be more consistent with the final pathologic diagnosis, as opposed to $2 \mathrm{D}$ techniques [41]. 

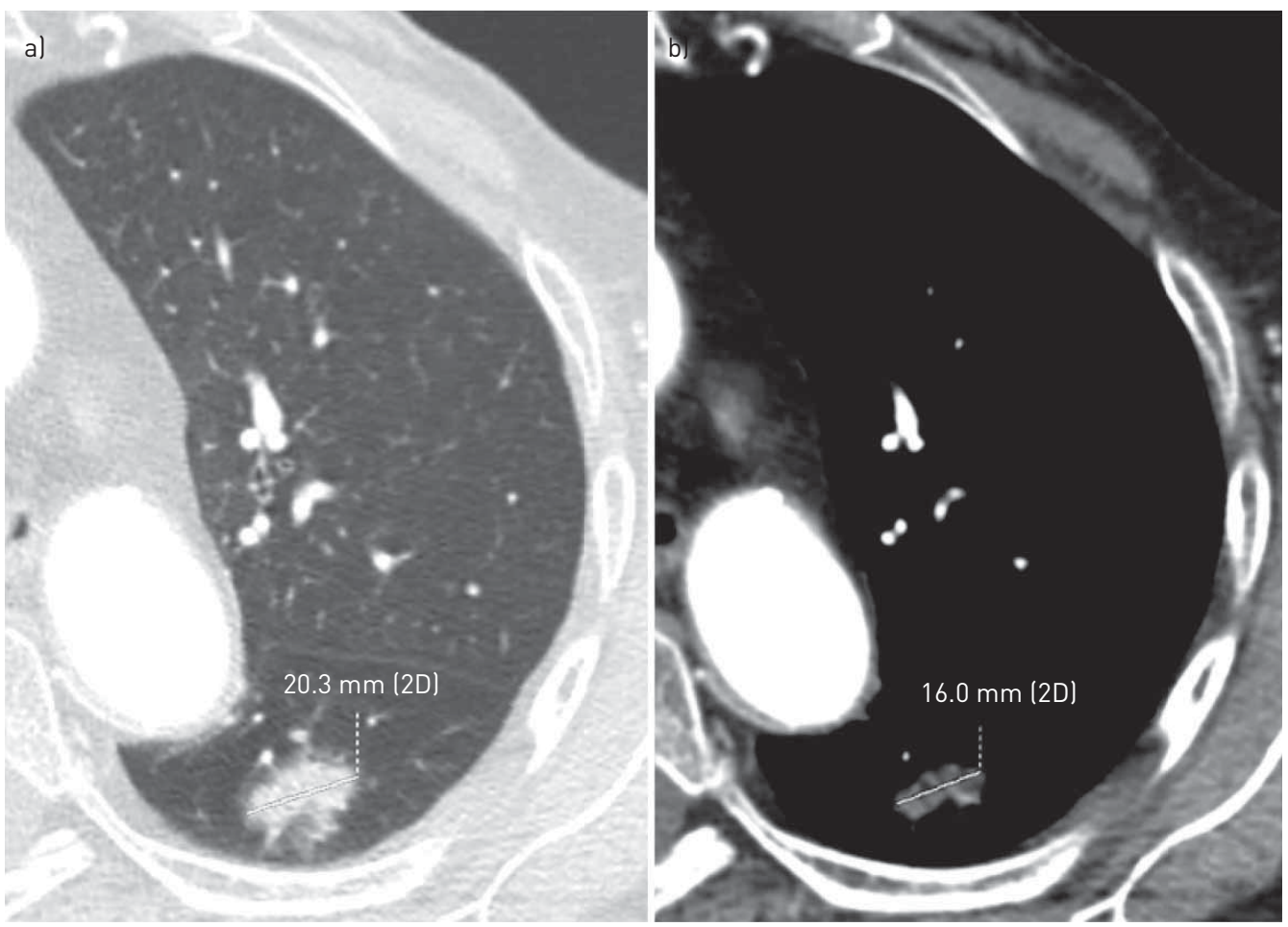

FIGURE 2 Disagreement in measuring the solid portion of a part-solid nodule when using different reconstruction algorithms and window settings. A part-solid nodule in the apical segment of left lower lobe is shown. a) By using a high-spatial frequency algorithm and the lung window, the measured maximum axial diameter of the solid portion of the nodule corresponds to $20.3 \mathrm{~mm}$; b) by using a smooth algorithm and the mediastinal window, the measured maximum axial diameter of the solid portion of the nodule corresponds to $16 \mathrm{~mm}$. 2D: two-dimensional.

In a preliminary experience with nodule 3D evaluation, Revel et al. [51] reported a maximum measurement error of $6.38 \%$ (upper limit of the $95 \%$ limit of acceptability) and underlined that a $6.38 \%$ increase in volume corresponds to a $2.1 \%$ increase in diameter (e.g. $0.1 \mathrm{~mm}$ and $0.2 \mathrm{~mm}$ for nodules measuring $5 \mathrm{~mm}$ and $10 \mathrm{~mm}$, respectively). Therefore, the precision of the $3 \mathrm{D}$ method can be considered to be much higher than that of the manual method of measuring diameter.

Moreover, high intra- and inter-reader agreement has been reported in the literature for volumetry (up to 0.99) [52-55], and volumetry performance was independent from the observer experience [55].

\section{Factors influencing nodule measurement variations}

The performance of $1 \mathrm{D}$ and $2 \mathrm{D}$ measurements depends mainly on nodule size, technical conditions and reading setting.

As regards size, major concerns exist in the measurement of small nodules. By using a field of view of $360 \mathrm{~mm}$ and an electronic matrix of $512 \times 512$, as is commonly applied in chest CT scan acquisition, the pixel dimension is $\sim 0.7 \mathrm{~mm}$ [56]. Therefore, a small difference in calliper positioning, even of a single pixel, could result in a significant difference in nodule size.

Regarding technical issues, nodules are better detected and characterised using thin and contiguous CT sections, as confirmed by results in the literature [2, 57-61]. It has been well established that contiguous thin-section CT scans reduce the partial volume effect that is responsible for errors in nodule margin delineation and in density recognition. In particular, it has been suggested that thin-section images increase sensitivity in detecting pGGNs and avoid the misinterpretation of solid nodules as SSNs [60]. Another parameter affecting accuracy in nodule measurement is the low tube current applied to perform CT scans, particularly in the screening programmes. The intrinsic increase in image noise of low-dose CT images may simulate the presence of a ground-glass opacity or may hide the margins of a pGGN, thus resulting in lesion misinterpretation and inaccurate measurement [60-62].

In addition, image reading settings may play an important role in assessing nodule size, particularly in the follow-up. Lower variability in lesion sizing has been reported when readers have the chance to consult 
previous measurements as compared to an "independent" reading session performed without any baseline measurement [63]. Furthermore, nodule size assessment performed during follow-up by the same radiologist and using automated software to compare images is helpful in reducing measurement variations, particularly as regards GGNs, for which subtle changes in size and density may be better underlined [64].

The accuracy and precision of 3D nodule volume measurement are influenced by multiple factors related to nodule/patient characteristics and technical issues.

Regarding nodule characteristics, volume overestimation of the small nodules due to the partial volume effect represents quite a challenge. It is a common imaging artefact when a limited spatial resolution is used to perform CT scans and, consequently, different tissues are included in the same pixel/voxel [50, 52, 65-69]. When attenuation value is not sufficient to distinguish nodule borders, segmentation errors could occur, as in the case of nonspherical or irregular lesions [41, 65, 68,70-72], as well as in juxtavascular or juxtapleural ones [72-74]. Reduced nodule attenuation, as in the case of SSNs, could also affect nodule segmentation when using the commonest threshold density technique, because of the low attenuation difference between nodule borders and the surrounding parenchyma [50]. Moreover, in PSNs the ground-glass component, usually peripheral, may hinder software detection of attenuation differences with the surrounding parenchyma, even for the solid portion [75]. Earlier studies described significantly higher errors of volumetry when evaluating SSNs in comparison to the solid nodules [76] and low correlation of volumetric assessment of the solid component (calculated as ratio of the solid component to the whole volume) with the histopathological classification [77]. Thanks to the development of specific software, volumetric measurement of SSNs has become accurate over the years with a successful segmentation of up to $97 \%$ of the nodules $[75,78-80]$. Similar results have been reported in the detection and segmentation of PSNs and, interestingly, a quantification of the solid component was related to pathological prognostic factors, such as lymphatic, vascular and pleural invasion [75, 81, 82].

As regards patient characteristics, cardiovascular motions affect volumetry because they are conveyed to lung parenchyma and determine changes in the volume of pulmonary nodules, especially the smallest ones [83]. Conflicting results are reported in the literature regarding the effect of respiratory phases on lung volume and, as a consequence, on the nodule volume measurement. Some authors showed an inverse relationship between inspiratory effort and nodule volume [84, 85], while others did not [65]. It should be kept in mind that CT volumetric measurements of SSNs, regarding both the ground-glass and solid components, showed a tendency to be larger than the histological counterpart, because of the different inflation state of the lung applied to a focal soft tumour $[49,78]$.

There are several technical factors affecting nodule volume estimation, such as section thickness [40, 68, 69, 86-89] and overlapping [90, 91], pitch mode [92], reconstruction algorithm [86, 89-91, 93-95] and intravenous contrast medium injection [95-97], as summarised in table 2. In addition, major technical concerns exist regarding nodule volumetry during follow-up. First, different performances are reported when using different scanner types [50, 86, 98]. Secondly, volumetry is affected by variability in the segmentation process due to differences in the method and software used. The automated method can introduce biases in volume measurements due to a different software performance, even though it has been demonstrated that it reduces observer variability $[113,114]$. Manual correction it is expected to act on these biases [55, 115]. Differences in volume estimation have been reported when using different software and different algorithms of correction of partial volume effect artefacts [57, 67, 116-118]. Therefore, it is advisable to perform nodule follow-up using the same scanner, technique and software package.

Another relevant issue is the potential influence of tube current on volumetry. Few experiences reported a low performance of volumetry due to tube current reduction [76, 99, 100]. A larger number of results derived from studies using newer generation scanners did not confirm the previous observations. Indeed, the introduction of iterative reconstructions, employed to increase image quality in favour of a further reduction of the effective radiation dose, demonstrated an even better performance compared to that of the traditionally used filtered-back projection reconstructions [101-112].

\section{Effect of measurement variations on nodule growth}

If we keep in mind the aforementioned exponential model of nodule growth, small change in nodule dimension may be clinically relevant. By using 1D and 2D methods small changes in nodule dimension may not be detected, resulting in a low sensitivity in identifying potential malignant lesions [42]. Furthermore, it has been demonstrated that growth assessment based on the maximum diameter measurement in noncalcified lung nodules, classified as positive at NLST, results in a moderate agreement among readers $(\kappa=0.55)$ with potential implications in patient management [119]. In the same way, 
TABLE 2 Technical factors that may affect volume measurement

\section{Effects on volumetry}

\section{Section thickness Overlapping reconstruction Pitch \\ Reconstruction algorithms}

\section{Intravenous contrast medium Scanner type \\ Low radiation dose Iterative reconstruction Manual versus semi/automated method}

Software and algorithms package
Volume overestimation with thick sections $[40,68,69,86-89]$

Volume overestimation with non-overlapping sections [90,91]

Low accuracy with high pitch mode [92]

Easier nodule sampling with high spatial frequency algorithms [86, 89, 91, 93]

Volume overestimation with high spatial frequency algorithms [90, 94, 95]

Volume overestimation [95-97]

Differences in volume estimation $[50,86,98]$

Higher accuracy with helical CT scanner [98]

Low accuracy $[76,99,100]$

Better performance [101-112]

Reduced observer variability with automated system $[113,114]$

Manual interaction reduces bias due to the software performance $[55,115]$

Differences in volume estimation $[57,67,116-118]$

CT: computed tomography.

relative errors have been reported when manually measuring 1D longest diameters according to the RECIST criteria to evaluate response to treatment of lung metastases [120]. These errors, when using 1D and $2 \mathrm{D}$ measurements, can lead to a big difference in estimating growth rate, considering the multiplier effect when volume and doubling time are estimated on the basis of diameter $[42,120]$.

Growth is a 3D phenomenon, therefore an asymmetrical growth could not be detected by using $1 \mathrm{D}$ or $2 \mathrm{D}$ methods, especially if it occurs in a different plane with respect from the axial one [41]. Intuitively, the direct assessment of nodule volume and VDT provides an accurate estimation of nodule growth [51]. Combined with lower uncertainty of measurements, the 3D method allows detection of changes even within a shorter period of time, resulting in a higher sensitivity of volume-based techniques in growth evaluation [26, 73] (figure 3). Estimations of nodule growth rates obtained from automated 3D volumetric measurements showed a good correlation with $2 \mathrm{D}$ diameter measurements, with a greater divergence for irregular lesions [70]. Since all the available data are included in the nodule volume definition and calculation, irregular nodules are evaluated with small magnitude errors and asymmetric growth could be reliably defined by using volumetric methods [41]. However, the reported volume measurement errors vary between $20 \%$ and $25 \%$, therefore a change in volume of $\geqslant 25 \%$ should be considered to define a significant growth $[2,33,121]$. In the screening setting, MARCHIANò et al. [122] reported similar values of repeatability, with the $95 \%$ confidence interval for the difference in measured volumes of $\pm 27 \%$.

\section{Subsolid nodules: special considerations}

When evaluating SSNs, nodule density provides major and additional information in terms of malignancy prediction.

While the proportion of ground-glass opacity was found to be a significant prognostic factor of less invasive cancer, the presence of a solid component corresponds to the pathological finding of tumour invasion and, therefore, represents a predictor of malignancy [2, 6]. Particularly in PSNs, a smaller solid portion has been described as an independent differentiator of a pre-invasive lesion from an invasive adenocarcinoma [123] and, moreover, the diameter of the solid component has a better correlation with patient prognosis than the whole-lesion diameter $[18,124]$. Finally, some typical radiological patterns, in terms of both nodule size and density, could be related to different histological categories described in the latest adenocarcinoma classification: the two premalignant (atypical adenomatous hyperplasia) and pre-invasive (adenocarcinoma in situ) lesions usually appear as pGGNs with a diameter of $<5 \mathrm{~mm}$ or $>5 \mathrm{~mm}$, respectively; minimally invasive adenocarcinoma as a PSN with a solid area $<5 \mathrm{~mm}$; and invasive adenocarcinoma as a larger PSN or solid nodule $[2,124,125]$.

Some studies have tried to identify a "threshold size" of different densitometric components in relation to nodule malignancy. Nodules with a ground-glass component of $>50 \%$ showed a significantly better prognosis [126]. In PSNs, LEE et al. [49] observed that a maximum diameter of the solid component of $\leqslant 3 \mathrm{~mm}$ was predictive of a pre-invasive or minimally invasive histology and two volumetric measurements (solid volume $\geqslant 1.5 \mathrm{~cm}^{3}$; percentage of solid volume $\geqslant 63 \%$ ) were found to be independent indicators 

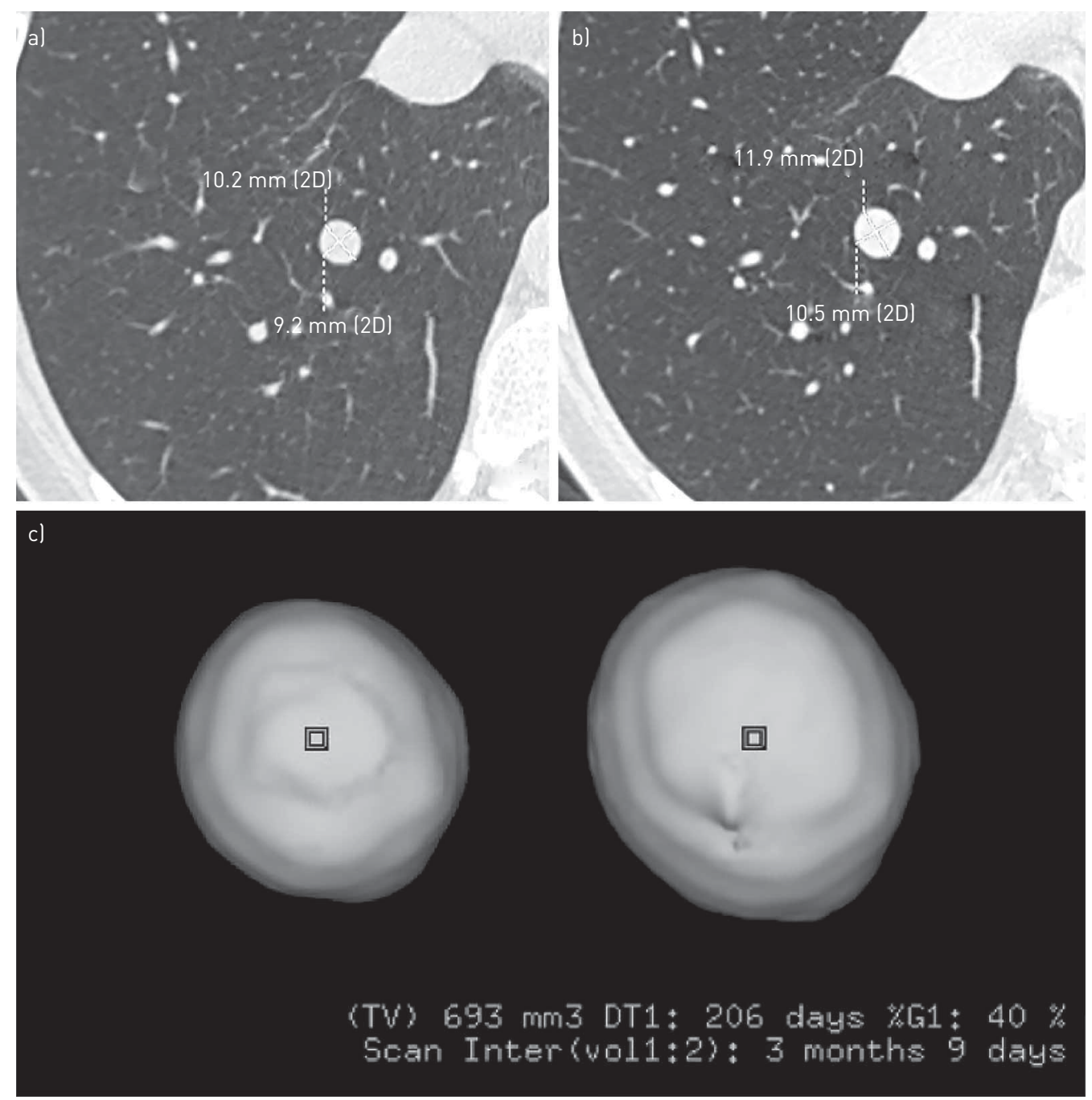

FIGURE 3 Volume evaluation during follow-up allows the detection of nodule growth over a shorter period of time compared to diameter estimation. al Computed tomography (CT) axial image shows the same nodule located in the right lower lobe as reported in figure 1c; b) a 3-month follow-up axial CT image demonstrates minimal change in nodule diameters; c) conversely, nodule volume calculation using a three-dimensional (3D) volumetric method demonstrates a significant increase in volume within the range of malignancy. Histopathology revealed a carcinoid tumour. 2D: two-dimensional; TV: total volume; DT: volume doubling time; \%G: volume increase; scan inter: scan interval. Squares in the nodule represent the starting points of the $3 \mathrm{D}$ analysis.

associated with increased likelihood of recurrence and/or death in patients with stage I adenocarcinoma [127]. Likewise, the ratio of the solid component to total tumour is related with tumour histology and therefore is a useful method of estimating prognosis [128, 129].

To reflect the changes in SSNs, not only in size but also in attenuation, another approach has been proposed, i.e. the estimation of the mass that integrates the nodule volume and density [130]. In a clinical evaluation, DE Hoop et al. [131], when applying nodule mass assessment (i.e. mean CT attenuation $\times$ volume) demonstrated a smaller measurement variability compared with diameter and volume and an earlier detection of nodule growth. Notably, the latter is due to a better capability of detecting the appearance or progression of a solid component in SSNs [131]. The usefulness of the system has been proven afterwards by other experimental studies [78, 81, 132] and used in the discrimination of histological subtypes in adenocarcinoma [133]. As for volumetric measurement, an existing interscan variability has been described for nodule mass assessment, and an increase in nodule mass of $30 \%$ has been regarded as a significant growth [134].

To corroborate the prognostic significance of nodule density in SSNs in terms of clinical decision making, the Fleischner Society recommendations for managing incidental SSNs categorised nodule risk on the basis of nodule density and not only on size and growth $[6,7]$. 
Similarly, the American College of Radiology published the Lung CT Screening Reporting and Data System (Lung-RADS) in 2014 [135], a scoring system that considered nodule density, in addition to size and growth, as relevant predictor of malignancy to categorise screening-detected lung nodules. The classification from 1 to $4 \mathrm{X}$ categories corresponds to an increasing risk of malignancy. Category $4 \mathrm{X}$ is assigned to nodules with additional imaging features requiring a more intensive diagnostic work-up [135].

The added value of the Lung-RADS category $4 \mathrm{X}$ in the differentiation of benign and malignant nodules has been evaluated for SSNs in a recent study by CHUNG et al. [136]. Six experienced chest radiologists were asked to analyse the characteristics of 374 SSNs in the NLST database that would have been classified as category 3, 4A, and $4 \mathrm{~B}$ according to the Lung-RADS system. The radiologists indicated which nodules were suspicious and that they would hence raise the Lung-RADS category to $4 \mathrm{X}$. In addition, the readers indicated which imaging characteristics made them upgrade the nodule to $4 \mathrm{X}$. Results demonstrated that the malignancy rate derived by adding morphological criteria (i.e. internal structure, presence of bullae, solid core characteristics, borders and surrounding tissue features) is superior to the risk assessed only on nodule type and size, with an average rate of malignancy of $53 \%$ with respect to the generic rate assigned by conventional Lung-RADS to the $4 \mathrm{X}$ category (>15\%) [136].

This observation emphasises the concept that the assessment of SSN characteristics by an expert radiologist outperforms the evaluation based only on nodule size and type in predicting malignancy.

\section{Integrating clinical and nodule characteristics: risk prediction models}

Several predictors of malignancy have been identified in a number of studies that reported multivariate analyses. When evaluating individuals with lung nodules, the probability of malignancy is estimated on the basis of patient-related clinical factors and nodule characteristics, including size [2, 4-6]. Among the clinical factors, older age, heavy current/former smoker, exposure to other inhaled carcinogens (asbestos, radon or uranium), as well as the presence of emphysema or fibrosis and family history of lung cancer have been demonstrated to be predictors of malignancy, as reported in the latest review of the Fleischner Society guidelines for nodule management [7]. As regards nodule morphological characteristics, besides small size, diffuse, central, laminated or popcorn calcifications, as well as fat tissue density and perifissural location have been recognised as indicative of benign lesions. In contrast, a large nodule diameter, or the evidence of nodule spiculation, upper lobe location, pleural indentation and VDT $<400$ days have been consistently identified as factors related to a higher risk of malignancy [2].

Some of these determinants have been included and tested in composite prediction models, developed with the scope to assist clinicians in the difficult task of nodule characterisation $[3,10,137]$. Currently the American College of Chest Physicians guidelines suggest using the Mayo Clinic prediction model based on patient categorisation into low $(>5 \%)$, intermediate $(5-65 \%)$ and high risk $(>65 \%)$ of malignancy [5], while the BTS guidelines suggest the use of the Brock and Herder models [2]. More recently, the Bayesian inference malignancy calculator model proved to be an accurate tool for characterising pulmonary nodules by guiding lesion-tailored diagnostic and interventional procedures during work-up [138].

In this context, it is worth mentioning that the accuracy and applicability of predictive models depend on the population in which they were derived and validated (e.g. screening, routine and oncology), according to differences in the prevalence of malignancy and in methods of evaluation.

\section{Size and follow-up recommendations}

The critical time for surveillance is the earliest point at which the nodule growth can be detected. Considering nodules detected in a screening programme, Kostis et al. [66] described nodule size at detection as a factor affecting the critical time for follow-up CT. Similarly, in the international guidelines for the management of indeterminate nodules, time surveillance is dependent on the initial nodule size; the bigger the nodule diameter the shorter the follow-up interval time [2, 4-7]. Despite the need for early diagnosis in cases of malignant nodules, it must be kept in mind that a higher accuracy of growth rate assessment and an improvement of malignancy risk evaluation with a longer interval time between the follow-up CT scans have been described in the literature $[6,24,70]$.

Some doubts remain regarding the duration of follow-up, not only because of the extremely long VDT of certain lung cancers, but also because some tumours (i.e. adenocarcinoma) showed a long period of stability before growing or even reducing in size during surveillance [23, 28, 139].

Nevertheless, the notion of a 2 -year stability implying benignity is widely accepted in common clinical practice, specifically for noncalcified solid pulmonary nodules, and the aforementioned results from the NELSON screening trial support such practice $[2,8]$. In contrast, a longer follow-up period is required for classifying for SSNs as benign with a reasonable certainty. 
In the latest revised Fleischner Society Guidelines [7], which take into consideration data from the major lung cancer screening projects in Europe and United States $[8,10,11,16,17,140]$ a new approach has been proposed for managing incidentally identified pulmonary nodules. For solid nodules, the minimum threshold of diameter requiring follow-up has been elevated to $6 \mathrm{~mm}$ in order to reduce false positives, and a follow-up time range has been introduced to reduce the number of examinations performed in the stable nodules. However, a longer period before the initial follow-up has been recommended for managing SSNs, because of their indolent nature when cancerous [7]. Reports in the current literature [17, 141] state that GGNs with diameter $\geqslant 6 \mathrm{~mm}$ should be followed-up for 5 years, with time scan intervals of 2 years, while PSN with a solid component $<6 \mathrm{~mm}$ should be evaluated annually for 5 years. In the case of PSNs with a solid component $\geqslant 6 \mathrm{~mm}$, after an initial follow-up, other nodule characteristics (such as morphological features and an eventual growth) as well as the clinical setting should guide further management [7].

\section{Conclusions}

With the diffusion of lung cancer screening programmes worldwide, the "database" of small pulmonary nodules has become huge. Furthermore, MDCT has dramatically increased the number of small-sized nodules identified on thin-section images. In this context, size and growth rate still represent pivotal factors for nodule characterisation, even though some limitations in evaluating pulmonary nodules when considering only their dimensions have been recognised. Firstly, there is no univocal method for measuring nodules (diameter, area, volume or mass). Secondly, intrinsic errors, which can determine variations in measurements and affect nodule growth assessment, do exist when using $1 \mathrm{D}, 2 \mathrm{D}$ and $3 \mathrm{D}$ methods. Finally, nodule CT attenuation has become a widely accepted significant determinant of prognosis over the past few years, specifically in SSNs. More recently, in these types of nodules, other morphological features (i.e. internal structure, presence of bullae, solid core characteristics, borders and surrounding tissue features) have been associated with an increased risk of malignancy.

Physicians should be aware that size and its change over time remain the most important factors determining nodule management, as stated in the currently used international guidelines, even though these factors should be evaluated in relation to other nodule characteristics, without overlooking the clinical context. Therefore, predictive models that take into account several factors have been proposed as a potential means to overcome the limitations of a size-based assessment of the malignancy risk for indeterminate pulmonary nodules.

\section{References}

1 Hansell DM, Bankier AA, MacMahon $\mathrm{H}$, et al. Fleischner Society: glossary of terms for thoracic imaging. Radiology 2008; 246: 697-722.

2 Callister ME, Baldwin DR, Akram AR, et al. British Thoracic Society guidelines for the investigation and management of pulmonary nodules. Thorax 2015; 70: Suppl. iil-ii54.

3 Swensen SJ, Silverstein MD, Ilstrup DM, et al. The probability of malignancy in solitary pulmonary nodules. Application to small radiologically indeterminate nodules. Arch Intern Med 1997; 157: 849-855.

4 MacMahon H, Austin JH, Gamsu G, et al. Guidelines for management of small pulmonary nodules detected on CT scans: a statement from the Fleischner Society. Radiology 2005; 237: 395-400.

5 Gould MK, Donington J, Lynch WR, et al. Evaluation of individuals with pulmonary nodules: when is it lung cancer? Diagnosis and management of lung cancer, 3rd ed: American College of Chest Physicians evidence-based clinical practice guidelines. Chest 2013; 143: Suppl. e93S-e120S.

6 Naidich DP, Bankier AA, MacMahon $\mathrm{H}$, et al. Recommendations for the management of subsolid pulmonary nodules detected at CT: a statement from the Fleischner Society. Radiology 2013; 266: 304-317.

7 MacMahon H, Naidich DP, Goo JM, et al. Guidelines for management of incidental pulmonary nodules detected on CT images: from the Fleischner Society 2017. Radiology 2017; 284: 228-243.

8 Horeweg N, van Rosmalen J, Heuvelmans MA, et al. Lung cancer probability in patients with CT-detected pulmonary nodules: a prespecified analysis of data from the NELSON trial of low-dose CT screening. Lancet Oncol 2014; 15: 1332-1341.

9 Wahidi MM, Govert JA, Goudar RK, et al. Evidence for the treatment of patients with pulmonary nodules: when is it lung cancer? ACCP evidence-based clinical practice guidelines (2nd edition). Chest 2007; 132: Suppl., 94S-107S.

10 McWilliams A, Tammemagi MC, Mayo JR, et al. Probability of cancer in pulmonary nodules detected on first screening CT. N Engl J Med 2013; 369: 910-919.

11 National Lung Screening Trial Research Team, Aberle DR, Adams AM, et al. Reduced lung-cancer mortality with low-dose computed tomographic screening. N Engl J Med 2011; 365: 395-409.

12 National Lung Screening Trial Research Team, Church TR, Black WC, et al. Results of initial low-dose computed tomographic screening for lung cancer. N Engl J Med 2013; 368: 1980-1991.

13 Henschke CI, McCauley DI, Yankelevitz DF, et al. Early Lung Cancer Action Project: overall design and findings from baseline screening. Lancet 1999; 354: 99-105.

14 Henschke CI, Yankelevitz DF, Mirtcheva R, et al. CT screening for lung cancer: frequency and significance of part-solid and nonsolid nodules. AJR Am J Roentgenol 2002; 178: 1053-1057.

15 Swensen SJ, Jett JR, Hartman TE, et al. Lung cancer screening with CT: Mayo Clinic experience. Radiology 2003; 226: $756-761$. 
Yankelevitz DF, Yip R, Smith JP, et al. CT screening for lung cancer: nonsolid nodules in baseline and annual repeat rounds. Radiology 2015; 277: 555-564.

17 Henschke CI, Yip R, Smith JP, et al. CT screening for lung cancer: part-solid nodules in baseline and annual repeat rounds. AJR Am J Roentgenol 2016; 207: 1176-1184.

18 Nakamura S, Fukui T, Taniguchi T, et al. Prognostic impact of tumor size eliminating the ground glass opacity component: modified clinical $\mathrm{T}$ descriptors of the tumor, node, metastasis classification of lung cancer. $J$ Thorac Oncol 2013; 8: 1551-1557.

19 Travis WD, Asamura H, Bankier AA, et al. The IASLC lung cancer staging project: proposals for coding T categories for subsolid nodules and assessment of tumor size in part-solid tumors in the forthcoming eighth edition of the TNM classification of lung cancer. J Thorac Oncol 2016; 11: 1204-1223.

20 Yankelevitz DF, Gupta R, Zhao B, et al. Small pulmonary nodules: evaluation with repeat CT - preliminary experience. Radiology 1999; 212: 561-566.

21 Zhao YR, Heuvelmans MA, Dorrius MD, et al. Features of resolving and nonresolving indeterminate pulmonary nodules at follow-up CT: the NELSON study. Radiology 2014; 270: 872-879.

22 Collins VP, Loeffler RK, Tivey H. Observations on growth rates of human tumors. Am J Roentgenol Radium Ther Nucl Med 1956; 76: 988-1000.

23 Lindell RM, Hartman TE, Swensen SJ, et al. 5-year lung cancer screening experience: growth curves of 18 lung cancers compared to histologic type, CT attenuation, stage, survival, and size. Chest 2009; 136: 1586-1595.

24 Xu DM, van der Zaag-Loonen HJ, Oudkerk M, et al. Smooth or attached solid indeterminate nodules detected at baseline CT screening in the NELSON study: cancer risk during 1 year of follow-up. Radiology 2009; 250: 264-272.

25 Henschke CI, Yankelevitz DF, Yip R, et al. Lung cancers diagnosed at annual CT screening: volume doubling times. Radiology 2012; 263: 578-583.

26 Revel MP, Merlin A, Peyrard S, et al. Software volumetric evaluation of doubling times for differentiating benign versus malignant pulmonary nodules. AJR Am J Roentgenol 2006; 187: 135-142.

27 Hasegawa M, Sone S, Takashima S, et al. Growth rate of small lung cancers detected on mass CT screening. $\mathrm{Br} J$ Radiol 2000; 73: 1252-1259.

28 Jennings SG, Winer-Muram HT, Tann M, et al. Distribution of stage I lung cancer growth rates determined with serial volumetric CT measurements. Radiology 2006; 241: 554-563.

29 Wilson DO, Ryan A, Fuhrman C, et al. Doubling times and CT screen-detected lung cancers in the Pittsburgh Lung Screening Study. Am J Respir Crit Care Med 2012; 185: 85-89.

30 Winer-Muram HT, Jennings SG, Tarver RD, et al. Volumetric growth rate of stage I lung cancer prior to treatment: serial CT scanning. Radiology 2002; 223: 798-805.

31 Song YS, Park CM, Park SJ, et al. Volume and mass doubling times of persistent pulmonary subsolid nodules detected in patients without known malignancy. Radiology 2014; 273: 276-284.

$32 \mathrm{Xu} \mathrm{DM}$, Gietema $\mathrm{H}$, de Koning $\mathrm{H}$, et al. Nodule management protocol of the NELSON randomised lung cancer screening trial. Lung Cancer 2006; 54: 177-184.

33 Sullivan DC, Obuchowski NA, Kessler LG, et al. Metrology standards for quantitative imaging biomarkers. Radiology 2015; 277: 813-825.

34 Jennings SG, Winer-Muram HT, Tarver RD, et al. Lung tumor growth: assessment with CT - comparison of diameter and cross-sectional area with volume measurements. Radiology 2004; 231: 866-871.

35 Petrick N, Kim HJ, Clunie D, et al. Comparison of 1D, 2D, and 3D nodule sizing methods by radiologists for spherical and complex nodules on thoracic CT phantom images. Acad Radiol 2014; 21: 30-40.

36 Mehta HJ, Ravenel JG, Shaftman SR, et al. The utility of nodule volume in the context of malignancy prediction for small pulmonary nodules. Chest 2014; 145: 464-472.

37 Heuvelmans MA, Vliegenthart R, Oudkerk M. Contributions of the European trials (European randomized screening group) in computed tomography lung cancer screening. $J$ Thorac Imaging 2015; 30: 101-107.

38 Goo JM. Computer-aided detection of lung nodules on chest CT: issues to be solved before clinical use. Korean J Radiol 2005; 6: 62-63.

39 Jaffe CC. Measures of response: RECIST, WHO, and new alternatives. J Clin Oncol 2006; 24: 3245-3251.

40 Zhao B, Tan Y, Bell DJ, et al. Exploring intra- and inter-reader variability in uni-dimensional, bi-dimensional, and volumetric measurements of solid tumors on CT scans reconstructed at different slice intervals. Eur J Radiol 2013; 82: 959-968.

41 Yankelevitz DF, Reeves AP, Kostis WJ, et al. Small pulmonary nodules: volumetrically determined growth rates based on CT evaluation. Radiology 2000; 217: 251-256.

42 Revel MP, Bissery A, Bienvenu M, et al. Are two-dimensional CT measurements of small noncalcified pulmonary nodules reliable? Radiology 2004; 231: 453-458.

43 Gierada DDS, Pilgram TKT, Ford M, et al. Lung cancer: interobserver agreement on interpretation of pulmonary findings at low-dose CT screening. Radiology 2008; 246: 265-272.

44 Bankier AA, MacMahon H, Goo JM, et al. Recommendations for measuring pulmonary nodules at CT: a statement from the Fleischner Society. Radiology 2017; 285: 584-600.

45 van Riel SI, Sánchez CI, Bankier AA, et al. Observer variability of classification of pulmonary nodules on low-dose CT imaging and its effect on nodule management. Radiology 2015; 277: 863-871.

46 Kim H, Park CM, Song YS, et al. Measurement variability of persistent pulmonary subsolid nodules on same-day repeat CT: what is the threshold to determine true nodule growth during follow-up? PLoS One 2016; 11: e0148853.

47 Penn A, Ma M, Chou BB, et al. Inter-reader variability when applying the 2013 Fleischner guidelines for potential solitary subsolid lung nodules. Acta Radiol 2015; 56: 1180-1186.

48 Yoo RE, Goo JM, Hwang EJ, et al. Retrospective assessment of interobserver agreement and accuracy in classifications and measurements in subsolid nodules with solid components less than $8 \mathrm{~mm}$ : which window setting is better? Eur Radiol 2017; 27: 1369-1376.

49 Lee KH, Goo JM, Park SJ, et al. Correlation between the size of the solid component on thin-section CT and the invasive component on pathology in small lung adenocarcinomas manifesting as ground-glass nodules. $J$ Thorac Oncol 2014; 9: 74-82. 
Gavrielides M, Kinnard LM, Myers KJ, et al. Noncalcified lung nodules: volumetric assessment with thoracic CT. Radiology 2009; 251: 26-37.

Revel M-P, Lefort C, Bissery A, et al. Pulmonary nodules: preliminary experience with three-dimensional evaluation. Radiology 2004; 231: 459-466.

Goodman LR, Gulsun M, Washington L, et al. Inherent variability of CT lung nodule measurements in vivo using semiautomated volumetric measurements. AJR Am J Roentgenol 2006; 186: 989-994.

Gietema HA, Wang $\mathrm{Y}, \mathrm{Xu} \mathrm{D}$, et al. Pulmonary nodules detected at lung cancer screening: interobserver variability of semiautomated volume measurements. Radiology 2006; 241: 251-257.

Wormanns D, Kohl G, Klotz E, et al. Volumetric measurements of pulmonary nodules at multi-row detector CT: in vivo reproducibility. Eur Radiol 2004; 14: 86-92.

Bolte $\mathrm{H}$, Jahnke $\mathrm{T}$, Schäfer FK, et al. Interobserver-variability of lung nodule volumetry considering different segmentation algorithms and observer training levels. Eur J Radiol 2007; 64: 285-295.

Goodsitt MM, Chan H, Way TW, et al. Accuracy of the CT numbers of simulated lung nodules images with multi-detector CT scanners. Med Phys 2009; 33: 3006-3017.

Zhao YR, van Ooijen PM, Dorrius MD, et al. Comparison of three software systems for semi-automatic volumetry of pulmonary nodules on baseline and follow-up CT examinations. Acta Radiol 2014; 55: 691-698.

Sinsuat M, Saita S, Kawata Y, et al. Influence of slice thickness on diagnoses of pulmonary nodules using low-dose CT: potential dependence of detection and diagnostic agreement on features and location of nodule. Acad Radiol 2011; 18: 594-604.

Lee HY, Goo JM, Lee HJ, et al. Usefulness of concurrent reading using thin-section and thick-section CT images in subcentimetre solitary pulmonary nodules. Clin Radiol 2009; 64: 127-132.

Park CM, Goo JM, Lee HJ, et al. Nodular ground-glass opacity at thin-section CT: histologic correlation and evaluation of change at follow-up. Radiographics 2007; 27: 391-408.

Goo JM, Park CM, Lee HJ. Ground-glass nodules on chest CT as imaging biomarkers in the management of lung adenocarcinoma. AJR Am J Roentgenol 2011; 196: 533-543.

Funama Y, Awai K, Liu D, et al. Detection of nodules showing ground-glass opacity in the lungs at low-dose multidetector computed tomography: phantom and clinical study. J Comput Assist Tomogr 2009; 33: 49-53. patient data sets acquired under "no change" conditions. Transl Oncol 2015; 8: 55-64.

Staring M, Pluim JP, de Hoop B, et al. Image subtraction facilitates assessment of volume and density change in ground-glass opacities in chest CT. Invest Radiol 2009; 44: 61-66.

Gietema HA, Schaefer-Prokop CM, Mali WP, et al. Pulmonary nodules: interscan variability of semiautomated volume measurements with multisection CT - influence of inspiration level, nodule size, and segmentation performance. Radiology 2007; 245: 888-894.

Kostis WJ, Yankelevitz DF, Reeves AP, et al. Small pulmonary nodules: reproducibility of three-dimensional volumetric measurement and estimation of time to follow-up CT. Radiology 2004; 231: 446-452.

de Hoop B, Gietema H, van Ginneken B, et al. A comparison of six software packages for evaluation of solid lung nodules using semi-automated volumetry: what is the minimum increase in size to detect growth in repeated CT examinations. Eur Radiol 2009; 19: 800-808.

Petrou M, Quint LE, Nan B, et al. Pulmonary nodule volumetric measurement variability as a function of CT slice thickness and nodule morphology. AJR Am J Roentgenol 2007; 188: 306-312.

Winer-Muram HT, Jennings SG, Meyer CA, et al. Effect of varying CT section width on volumetric measurement of lung tumors and application of compensatory equations. Radiology 2003; 229: 184-194.

Korst RJ, Lee BE, Krinsky GA, et al. The utility of automated volumetric growth analysis in a dedicated pulmonary nodule clinic. J Thorac Cardiovasc Surg 2011; 142: 372-377.

Xie X, Willemink MJ, de Jong PA, et al. Small irregular pulmonary nodules in low-dose CT: observer detection sensitivity and volumetry accuracy. AJR Am J Roentgenol 2014; 202: W202-W209.

Wang Y, van Klaveren RJ, van der Zaag-Loonen HJ, et al. Effect of nodule characteristics on variability of semiautomated volume measurements in pulmonary nodules detected in a lung cancer screening program. Radiology 2008; 248: 625-631.

Ko JP, Berman EJ, Kaur M, et al. Pulmonary nodules: growth rate assessment in patients by using serial CT and three-dimensional volumetry. Radiology 2012; 262: 662-671.

Ko JP, Marcus R, Bomsztyk E, et al. Effect of blood vessels on measurement of nodule volume in a chest phantom. Radiology 2006; 239: 79-85.

Benzakoun J, Bommart S, Coste J, et al. Computer-aided diagnosis (CAD) of subsolid nodules: evaluation of a commercial CAD system. Eur J Radiol 2016; 85: 1728-1734.

study. Radiology 2003; 228: 864-870.

Sumikawa $\mathrm{H}$, Johkoh $\mathrm{T}$, Nagareda $\mathrm{T}$, et al. Pulmonary adenocarcinomas with ground-glass attenuation on thin-section CT: quantification by three-dimensional image analyzing method. Eur J Radiol 2008; 65: 104-111.

Scholten ET, de Hoop B, Jacobs C, et al. Semi-automatic quantification of subsolid pulmonary nodules: comparison with manual measurements. PLoS One 2013; 8: e80249.

Oda S, Awai K, Murao K, et al. Computer-aided volumetry of pulmonary nodules exhibiting ground-glass opacity at MDCT. AJR Am J Roentgenol 2010; 194: 398-406.

Park CM, Goo JM, Lee HJ, et al. Persistent pure ground-glass nodules in the lung: interscan variability of semiautomated volume and attenuation measurements. AJR Am J Roentgenol 2010; 195: 408-414.

Scholten ET, Jacobs C, van Ginneken B, et al. Detection and quantification of the solid component in pulmonary subsolid nodules by semiautomatic segmentation. Eur Radiol 2015; 25: 488-496.

Yanagawa M, Tanaka Y, Kusumoto M, et al. Automated assessment of malignant degree of small peripheral adenocarcinomas using volumetric CT data: correlation with pathologic prognostic factors. Lung Cancer 2010; 70: 286-294.

Boll DT, Gilkeson RC, Fleiter TR, et al. Volumetric assessment of pulmonary nodules with ECG-gated MDCT. AJR Am J Roentgenol 2004; 183: 1217-1223. 
Petkovska I, Brown MS, Goldin JG, et al. The effect of lung volume on nodule size on CT. Acad Radiol 2007; 14: 476-485.

Goo JM, Kim KG, Gierada DS, et al. Volumetric measurements of lung nodules with multi-detector row CT: effect of changes in lung volume. Korean J Radiol 2006; 7: 243-248.

Das M, Ley-Zaporozhan J, Gietema HA, et al. Accuracy of automated volumetry of pulmonary nodules across different multislice CT scanners. Eur Radiol 2007; 17: 1979-1984.

Larici AR, Storto ML, Torge M, et al. Automated volumetry of pulmonary nodules on multidetector CT: influence of slice thickness, reconstruction algorithm and tube current. Preliminary results. Radiol Med 2008; 113: 29-42.

Nietert PJ, Ravenel JG, Leue WM, et al. Imprecision in automated volume measurements of pulmonary nodules and its effect on the level of uncertainty in volume doubling time estimation. Chest 2009; 135: 1580-1587.

Ravenel JG, Leue WM, Nietert PJ, et al. Pulmonary nodule volume: effects of reconstruction parameters on automated measurements - a phantom study. Radiology 2008; 247: 400-408.

Honda O, Sumikawa $\mathrm{H}$, Johkoh T, et al. Computer-assisted lung nodule volumetry from multi-detector row CT: influence of image reconstruction parameters. Eur J Radiol 2007; 62: 106-113.

Gavrielides MA, Zeng R, Myers KJ, et al. Benefit of overlapping reconstruction for improving the quantitative assessment of CT lung nodule volume. Acad Radiol 2013; 20: 173-180.

Hwang SH, Oh YW, Ham SY, et al. Effect of the high-pitch mode in dual-source computed tomography on the accuracy of three-dimensional volumetry of solid pulmonary nodules: a phantom study. Korean J Radiol 2015; 16: 641-647.

Goo JM, Tongdee T, Tongdee R, et al. Volumetric measurement of synthetic lung nodules with multi-detector row CT: effect of various image reconstruction parameters and segmentation thresholds on measurement accuracy. Radiology 2005; 235: 850-856.

Wang Y, de Bock GH, van Klaveren RJ, et al. Volumetric measurement of pulmonary nodules at low-dose chest CT: effect of reconstruction setting on measurement variability. Eur Radiol 2010; 20: 1180-1187.

Honda O, Johkoh T, Sumikawa $\mathrm{H}$, et al. Pulmonary nodules: 3D volumetric measurement with multidetector CT - effect of intravenous contrast medium. Radiology 2007; 245: 881-887.

De Jong PA, Leiner T, Lammers JWJ, et al. Can low-dose unenhanced chest CT be used for follow-up of lung nodules? AJR Am J Roentgenol 2012; 199: 777-780.

Rampinelli C, Raimondi S, Padrenostro M, et al. Pulmonary nodules: contrast-enhanced volumetric variation at different CT scan delays. AJR Am J Roentgenol 2010; 195: 149-154.

Das M, Mühlenbruch G, Katoh M, et al. Automated volumetry of solid pulmonary nodules in a phantom: accuracy across different CT scanner technologies. Invest Radiol 2007; 42: 297-302.

Rampinelli C, De Fiori E, Raimondi S, et al. In vivo repeatability of automated volume calculations of small pulmonary nodules with CT. AJR Am J Roentgenol 2009; 192: 1657-1661.

Linning E, Daqing M. Volumetric measurement pulmonary ground-glass opacity nodules with multi-detector CT: effect of various tube current on measurement accuracy - a chest CT phantom study. Acad Radiol 2009; 16: 934-939.

Young S, Kim HJ, Ko MM, et al. Variability in CT lung-nodule volumetry: effects of dose reduction and reconstruction methods. Med Phys 2015; 42: 2679-2678.

Willemink MJ, Leiner T, Budde RP, et al. Systematic error in lung nodule volumetry: effect of iterative reconstruction versus filtered back projection at different CT parameters. AJR Am J Roentgenol 2012; 199: 1241-1246.

Wielpütz MO, Wroblewski J, Lederlin M, et al. Computer-aided detection of artificial pulmonary nodules using an ex vivo lung phantom: influence of exposure parameters and iterative reconstruction. Eur J Radiol 2015; 84: 1005-1011.

Gartenschläger M, Schweden F, Gast K, et al. Pulmonary nodules: detection with low-dose vs conventional-dose spiral CT. Eur Radiol 1998; 8: 609-614.

Xie X, Willemink MJ, Zhao $\mathrm{Y}$, et al. Inter-and intrascanner variability of pulmonary nodule volumetry on low-dose 64-row CT: an anthropomorphic phantom study. Br J Radiol 2013; 86: 17-19.

Christe A, Torrente JC, Lin M, et al. CT screening and follow-up of lung nodules: effects of tube current-time setting and nodule size and density on detectability and of tube current-time setting on apparent size. AJR Am J Roentgenol 2011; 197: 623-630.

Karabulut N, Törü M, Gelebek V, et al. Comparison of low-dose and standard-dose helical CT in the evaluation of pulmonary nodules. Eur Radiol 2002; 12: 2764-2769.

Hein PA, Romano VC, Rogalla P, et al. Variability of semiautomated lung nodule volumetry on ultralow-dose CT: comparison with nodule volumetry on standard-dose CT. J Digit Imaging 2010; 23: 8-17.

Scholten ET, Jacobs C, van Ginneken B, et al. Computer-aided segmentation and volumetry of artificial ground-glass nodules at chest CT. AJR Am J Roentgenol 2013; 201: 295-300.

Siegelman JW, Supanich MP, Gavrielides MA. Pulmonary nodules with ground-glass opacity can be reliably measured with low-dose techniques regardless of iterative reconstruction: results of a phantom study. AJR Am J Roentgenol 2015; 204: 1242-1247.

11 Kim H, Park CM, Kim SH, et al. Persistent pulmonary subsolid nodules: model-based iterative reconstruction for nodule classification and measurement variability on low-dose CT. Eur Radiol 2014; 24: 2700-2708.

Sakai N, Yabuuchi H, Kondo M, et al. Volumetric measurement of artificial pure ground-glass nodules at low-dose CT: comparisons between hybrid iterative reconstruction and filtered back projection. Eur J Radiol 2015; 84: 2654-2662.

Meyer CR, Johnson TD, McLennan G, et al. Evaluation of lung MDCT nodule annotation across radiologists and methods. Acad Radiol 2006; 13: 1254-1265.

Xie X, Zhao Y, Snijder RA, et al. Sensitivity and accuracy of volumetry of pulmonary nodules on low-dose 16and 64-row multi-detector CT: an anthropomorphic phantom study. Eur Radiol 2013; 23: 139-147.

Bolte H, Riedel C, Müller-Hülsbeck S, et al. Precision of computer-aided volumetry of artificial small solid pulmonary nodules in ex vivo porcine lungs. Br J Radiol 2007; 80: 414-421. 
116 Ashraf H, De Hoop B, Shaker SB, et al. Lung nodule volumetry: segmentation algorithms within the same software package cannot be used interchangeably. Eur Radiol 2010; 20: 1878-1885.

117 Rinaldi MF, Bartalena T, Braccaioli L, et al. Three-dimensional analysis of pulmonary nodules: variability of semiautomated volume measurements between different versions of the same software. Radiol Med 2010; 115: 403-412.

118 Athelogou M, Kim HJ, Dima A, et al. Algorithm variability in the estimation of lung nodule volume from phantom CT scans: results of the QIBA 3A public challenge. Acad Radiol 2016; 23: 940-952.

119 Singh S, Pinsky P, Fineberg NS, et al. Evaluation of reader variability in the interpretation of follow-up CT scans at lung cancer screening. Radiology 2011; 259: 263-270.

120 Marten K, Auer F, Schmidt S, et al. Inadequacy of manual measurements compared to automated CT volumetry in assessment of treatment response of pulmonary metastases using RECIST criteria. Eur Radiol 2006; 16: $781-790$.

121 van Klaveren RJ, Oudkerk M, Prokop M, et al. Management of lung nodules detected by volume CT scanning. $N$ Engl J Med 2009; 361: 2221-2229.

122 Marchianò A, Calabrò E, Civelli E, et al. Pulmonary nodules: volume repeatability at multidetector CT lung cancer screening. Radiology 2009; 251: 919-925.

123 Lee SM, Park CM, Goo JM, et al. Invasive pulmonary adenocarcinomas versus preinvasive lesions appearing as ground-glass nodules: differentiation by using CT features. Radiology 2013; 268: 265-273.

124 Hwang EJ, Park CM, Ryu Y, et al. Pulmonary adenocarcinomas appearing as part-solid ground-glass nodules: is measuring solid component size a better prognostic indicator? Eur Radiol 2015; 25: 558-567.

125 Travis WD, Brambilla E, Noguchi M, et al. International Association for the Study of Lung Cancer/American Thoracic Society/European Respiratory Society international multidisciplinary classification of lung adenocarcinoma. J Thorac Oncol 2011; 6: 244-285.

126 Aoki T, Tomoda Y, Watanabe H, et al. Peripheral lung adenocarcinoma: correlation of thin-section CT findings with histologic prognostic factors and survival. Radiology 2001; 220: 803-809.

127 Yanagawa M, Tanaka Y, Leung AN, et al. Prognostic importance of volumetric measurements in stage I lung adenocarcinoma. Radiology 2014; 272: 557-567.

128 Yanagawa M, Kuriyama K, Kunitomi Y, et al. One-dimensional quantitative evaluation of peripheral lung adenocarcinoma with or without ground-glass opacity on thin-section CT images using profile curves. $\mathrm{Br} J$ Radiol 2009; 82: 532-540.

129 Suzuki K, Koike T, Asakawa T, et al. A prospective radiological study of thin-section computed tomography to predict pathological noninvasiveness in peripheral clinical IA lung cancer (Japan Clinical Oncology Group 0201). J Thorac Oncol 2011; 6: 751-756.

130 Lee KW, Im JG, Kim TJ, et al. A new method of measuring the amount of soft tissue in pulmonary ground-glass opacity nodules: a phantom study. Korean J Radiol 2008; 9: 219-225.

131 de Hoop B, Gietema H, van de Vorst S, et al. Pulmonary ground-glass nodules: increase in mass as an early indicator of growth. Radiology 2010; 255: 199-206.

132 Kim H, Park CM, Woo S, et al. Pure and part-solid pulmonary ground-glass nodules: measurement variability of volume and mass in nodules with a solid portion less than or equal to $5 \mathrm{~mm}$. Radiology 2013; 269: 585-593.

133 Ko JP, Suh J, Ibidapo O, et al. Lung adenocarcinoma: correlation of quantitative CT findings with pathologic findings. Radiology 2016; 280: 931-939.

134 Scholten ET, de Jong PA, Jacobs C, et al. Interscan variation of semi-automated volumetry of subsolid pulmonary nodules. Eur Radiol 2015; 25: 1040-1047.

135 American College of Radiology. Lung CT Screening Reporting and Data System (Lung-RADS). www.acr.org/ Quality-Safety/Resources/LungRADS Date last accessed May 12, 2016. Date last updated: April 28, 2014.

136 Chung K, Jacobs C, Scholten ET, et al. Lung-RADS category 4X: does it improve prediction of malignancy in subsolid nodules? Radiology 2017; 284: 264-271.

137 Gould MK, Ananth L, Barnett PG. A clinical model to estimate the pretest probability of lung cancer in patients with solitary pulmonary nodules. Chest 2007; 131: 383-388.

138 Soardi GA, Perandini S, Larici AR, et al. Multicentre external validation of the BIMC model for solid solitary pulmonary nodule malignancy prediction. Eur Radiol 2017; 27: 1929-1933.

139 Lindell RM, Hartman TE, Swensen SJ, et al. Five-year lung cancer screening experience: CT appearance, growth rate, location, and histologic features of 61 lung cancers. Radiology 2007; 242: 555-562.

140 Henschke CI, Yankelevitz DF, Libby DM, et al. Survival of patients with stage I lung cancer detected on CT screening. N Engl J Med 2006; 355: 1763-1771.

141 Kakinuma R, Muramatsu Y, Kusumoto M, et al. Solitary pure ground-glass nodules $5 \mathrm{~mm}$ or smaller: frequency of growth. Radiology 2015; 276: 873-882. 